\title{
Route to create large-area ordered polymeric nanochannel arrays
}

\author{
P. Müller-Buschbaum, ${ }^{\text {a) }}$ E. Bauer, E. Maurer, and K. Schlögl \\ TU München, Physik-Department, LS E13, James-Franck-Str.1, 85747 Garching, Germany \\ S. V. Roth and R. Gehrke \\ HASYLAB at DESY, Notkestr. 85, 22603 Hamburg, Germany
}

(Received 4 August 2005; accepted 11 January 2006; published online 23 February 2006)

\begin{abstract}
Depositing polymdimethylsiloxane (PDMS) from an isopropanol solution onto a glass slide surface by wiping with a fuzz-free wipe results in highly ordered structures. Dewetting of the highly diluted PDMS solution and evaporation of the solvent yields nanostructures. The structure is well characterized as polymer nanochannels, separated by a mean distance of $166 \mathrm{~nm}$. The mean height of the shallow channels is $3 \mathrm{~nm}$ only. The proof of having aligned structures on very large surface areas with a well defined orientation is performed with a very high resolution grazing incidence small angle x-ray scattering setup. (C) 2006 American Institute of Physics.
\end{abstract}

[DOI: $10.1063 / 1.2178402]$

Due to the numerous applications in microfluidics, electronic chip production, fabrication of bioanalytical assays, and sensors coatings the nanostructuring of polymer surfaces attracted large interest in basic research as well as in technology. Lithography based "top-down" techniques turned out to be powerful methods for the preparation of rather complex surface patterns. ${ }^{1,2}$ In contrast, self-assembly based "bottomup" techniques promise a cheap and easy access to structured surfaces. ${ }^{3-8}$ However, the preparation of special types of surface pattern, such as polymer nanochannels on top of solid supports, by the use of self-assembly is rather difficult. Polymer nanochannels are best described, by the alignment of polymeric material in parallel walls, without a polymeric connection in the perpendicular direction. ${ }^{9-11}$ Especially, the controlled structuring of large surface areas of many square millimeters remains challenging. Preparation routes based on diblock copolymers require the use of external fields and several special chemical steps to end up with highly aligned polymer nanochannels: ${ }^{12-15}$ With the help of the external field, the local lamellar order is expanded in a stripe-like pattern and a secondary chemical treatment is necessary to remove one block, yielding the nanochannels. Preparation routes based on optical interference result in large scale and extremely perfect surface gratings, ${ }^{16}$ but the special type of equipment required does not match the idea of a simple process. In the present investigation, we present an alternative unexplored way to prepare shallow polymer nanochannels on a solid support. Although not reaching the degree of perfection as compared to using diblock copolymers or optical interference, the advantage of the escribed route is its extreme simplicity and size scalability.

Polymdimethylsiloxane (PDMS) is deposited from an isopropanol solution onto a standard microscopy glass slide surface with surface roughness $<0.5 \mathrm{~nm}$. A $100 \%$-cellulosebased fuzz-free wipe ${ }^{17}$ is soaked with the highly diluted solution (concentration below $0.1 \mathrm{mg} / \mathrm{ml}$ ) and the deposition is realized by several successive wipings_using a contact pressure of $p=24 \pm 3 \mathrm{kPa}$-in parallel to the long axis of the glass slide, followed by the evaporation of the solvent isopropanol (climate controlled conditions at $T=26{ }^{\circ} \mathrm{C}$ ). During

${ }^{\text {a)} E l e c t r o n i c ~ m a i l: ~ m u e l l e r b @ p h . t u m . d e ~}$ this procedure the substrate is kept electrostatically isolated against the surrounding, thereby enabling a weak charging upon wiping. The highly diluted PDMS solution dewets the glass surface. ${ }^{18,19}$ Due to the very low concentration only a small amount of PDMS is transferred onto the glass surface. The evaporation of the solvent freezes-in the installed nanostructure initially induced by the interplay of orientational deposition and dewetting. As shown in the inset of Fig. 1, the resulting surface pattern remains invisible with optical microscopy, and cannot be distinguished from an untreated glass surface.

To overcome the optical resolution limit, the real space structure is characterized with atomic force microscopy (AFM) on a local scale. Figure 1 shows an AFM image with $15 \times 15 \mu \mathrm{m}^{2}$ scan range. The sample is tilted with respect to the scanning direction to reduce the influence of the scanning or data treatment (flattening) on the determination of the height of the polymer nanochannels. The PDMS is deposited in narrow long lines, which are highly aligned parallel with respect to each other. The mean height of the PDSM lines is $H=(3.0 \pm 0.5) \mathrm{nm}$ only. The mean distance between adjacent PDMS lines is $D=(166 \pm 9) \mathrm{nm}$ [see line scan from AFM data in Fig. 2(c)]. Because the number of polymer connections between neighboring lines is very limited, the structure shown in Fig. 1 fulfills the characteristics of polymer nanochannels. We emphasize, that the channels are extremely shallow which is difficult to achieve with other techniques. Without the mechanical wiping in one spatial direc-

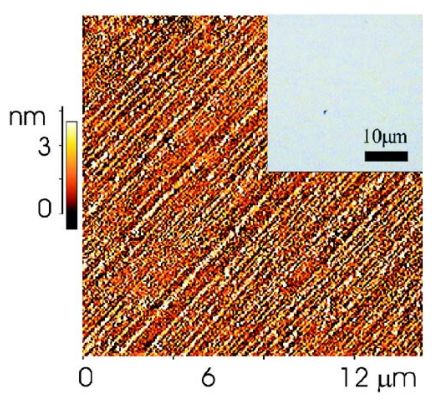

FIG. 1. (Color online) Real space structural data of the PDMS nanochannels: AFM topography data with $15 \times 15 \mu \mathrm{m}^{2}$ scan range and optical micrograph in the inset. 

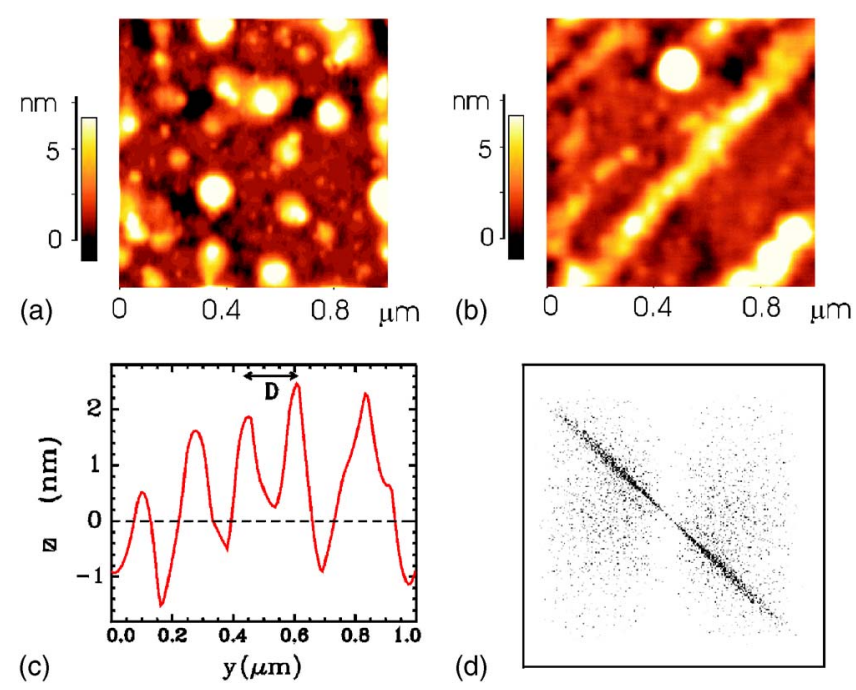

(d)

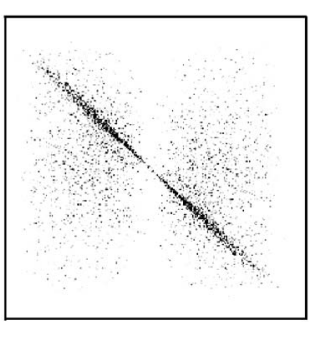

FIG. 2. (Color online) (a) For comparison, dewetting structure resulting without wiping and (b) zoomed-in AFM data with $1 \times 1 \mu \mathrm{m}^{2}$ scan range demonstrating imperfections of the PDMS nanochannels. (c) Representative line scan $(D=166 \mathrm{~nm})$ and (d) Fourier transformed calculated from the AFM data.

tion a common nanodewetting structure, well described by randomly distributed, pancake shaped PDMS islands, results [see Fig. 2(a).$^{20}$ The wiping process yields a symmetry breaking and induces a preferred orientation of the resulting structures in wiping direction.

The Fourier transform of the AFM data [see Fig. 2(d)] enables a statistical characterization of the surface pattern. The narrow stripes of intensity in Fourier space demonstrate the high degree of perfection of the alignment of PDMS into parallel lines and thus the strong anisotropy of the surface structure. However, only one broad intensity maximum is visible. A perfect surface grating would have resulted in numerous intensity maxima, well separated by the reciprocal grating period. The absence of higher order maxima is a clear indication of a relaxed order of the PDMS lines with respect to the distance between adjacent lines. Therefore, the preparation results rather in nanochannels than in a surface grating.

To further characterize the nanochannel array we focus on the imperfections of the polymer nanochannels. In Fig. 2(b) two typical types of defects are identified, namely along the direction and perpendicular to the nanochannels. For this purpose the AFM has zoomed in with $1 \times 1 \mu \mathrm{m}^{2}$ scan range. Along the direction of the PDMS lines, locally at a defect the line ruptured and the PDMS material coalescent into a drop ending the line. In addition, neighboring lines can have a distance significantly larger than the mean distance $D$. This is consistent with a broad distribution in $D$ and leads to the broad intensity maximum visible in Fig. 2(d).

To prove the presence of the polymer nanochannels over a very large surface area of many square millimeters, thus having prepared an ordered polymer nanochannel array, AFM is not suited. To overcome this problem, the surface pattern is investigated with grazing incidence small angle $\mathrm{X}$-ray scattering (GISAXS). ${ }^{21}$ GISAXS is the extension of SAXS in transmission geomtry to surfaces by combining SAXS and a grazing incidence setup. It enables the detection of polymer nanostructures with a high statistical significance. In addition, GISAXS allows for the detection of the orientation of the channels with respect to the edges of the glass Downloaded 30 Aug 2007 to 131.169.95.147. Redistribution subject substrate. The GISAXS experiments were performed at the BW4 beamline (HASYLAB, Hamburg) using a wavelength of $\lambda=0.138 \mathrm{~nm}$. For the current purpose an extremely small incident angle of $\alpha_{i}=0.01^{\circ}$ is selected. Therefore, on the one hand maximum surface sensitivity is achieved. On the other hand the full length of the glass slide $(86 \mathrm{~mm})$ has to be illuminated to prove the existence of the array. This is achieved due to the projection of the x-ray beam onto the sample surface. Because the incident angle is small as compared to the critical angles of the solid support (glass) and the polymer channels (PDMS), the penetration depth of the $\mathrm{X}$-ray beam perpendicular to the surface is limited to its smallest value. As a consequence, the setup has its highest surface sensitivity and enables the probing of structures with a few nanometers in height only.

In addition, for the first time, an ultrahigh resolution setup is operated to allow for the detection of the order and angular alignment of the PDMS nanochannels with respect to the underlying glass substrate. The beam divergence in and out of the plane of reflection is set by two narrow entrance cross slits to match the detector resolution. The nonspecular, diffuse as well as the specular intensity is recorded with a two-dimensional (2D) detector. At a very large sample-detector distance of $L_{\mathrm{SD}}=13.0 \mathrm{~m}$ a resolution of $2.75 \times 10^{-4} \mathrm{~nm}^{-1}$ is achieved. Consequently, the setup accesses lateral lengths from $35 \mathrm{~nm}$ up to theoretically $21 \mu \mathrm{m}$. The maximum accessible lateral length scale of this ultrahigh resolution setup has been confirmed by means of a Monte Carlo ray-tracing simulation of the beamline BW4. A beam stop in front of the detector is installed at the position of the specular peak to shield the detector. Due to the extremely small incident angle, the direct and the specularly reflected beam are located close to each other. In contrast, the Yoneda peak (at $\alpha_{f}=0.12^{\circ}$ ) is well separated in vertical direction from the beamstop (corresponding to $\alpha_{f}=0.0^{\circ}$ ) as well as in the direction parallel to the sample surface. ${ }^{21}$ Thus this setup allows for an easy separation of the specular and diffuse scattering.

The 2D intensity distribution can be understood as follows. The wave vector transfer is decomposed parallel and vertical to the sample surface. The two components of the parallel part are called $q_{y}$ and $q_{x}$, perpendicular and parallel to the incoming beam, respectively. The information encoded in a vertical slice (scattering vector vertical to the sample surface) probes the morphology vertical to the sample surface, essentially the height of the structures ${ }^{22}$ comparable to a reflection experiment. The intensity distribution in a horizontal slice, which is of most interest in our case due the extreme surface sensitivity at an incident angle $\alpha_{i}=0.01^{\circ}$, allows for extracting the lateral structure of the nanochannel array parallel to the sample surface: ${ }^{23}$ In our case we can focus on the distances present in the nanochannel array. This is the analogon to transmission SAXS probing distances perpendicular to the incoming beam. However, due to the grazing incidence geometry, refraction and reflection effects have to be taken into account. At the critical angle, the field amplitudes of incoming and totally reflected wave interfere, leading to an extreme structure sensitivity of the scattered signal at the critical angle. Hence, Fig. 3 shows a horizontal slice of the 2D intensity distribution cut at the critical angle of PDMS. The lateral component-parallel to the sample surface-of the wave vector is denoted $q_{y}$. Data of the sample with the polymer nanochannels are presented in comto AIP license or copyright, see http://apl.aip.org/apl/copyright.jsp 


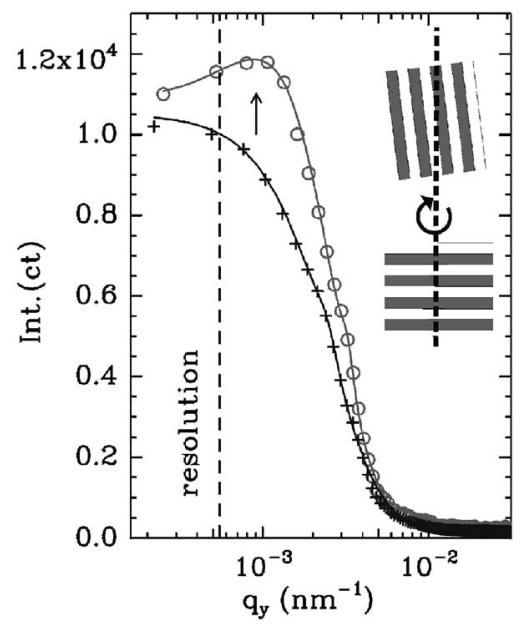

FIG. 3. GISAXS data of the glass surface with PDMS nanochannels on top (circles) in comparison with data of a bare glass surface (crosses). The arrow marks a position related to the structure factor of the tilted nanochannels. The dashed line indicates the resolution limit. The inset visualizes the rotation of the nanochannel array by $\omega$ with respect to the x-ray beam (dotted line).

parison with data of a bare glass surface. The solid lines are fits assuming structure and form factor as well as the resolution of the experimental setup. The statistical surface roughness of the glass is spatially uncorrelated at lengths within the resolution limit and thus the intensity decays monotonously as a function of $q_{y}$. In contrast, the presence of a well defined surface pattern results in the observation of a structure factor-like peak in the GISAXS data at a position $q_{y}^{p}$. From the fit the value of the related most prominent in-plane distance of $\Lambda=(7.0 \pm 0.1) \mu \mathrm{m}$ is determined. With $\quad \alpha_{i} \approx 0, \quad \operatorname{using}^{23,24} \quad q_{x}=(2 \pi / \lambda)\left[\cos \left(\alpha_{f}\right)-\cos \left(\alpha_{i}\right)\right]$ $\approx(2 \pi / \lambda)\left(1-\frac{1}{2} \alpha_{f}^{2}-1\right)$ one deduces the condition for the position of the zero-order maximum

$$
q_{y}^{p}+\frac{q_{x}}{\tan (\omega)}=0 \Leftrightarrow \tan (\omega)=-\frac{\pi}{\lambda} \frac{\alpha_{f}^{2}}{q_{y}^{p}},
$$

the orientation of the nanochannels in terms of the tilt angle $\omega$ with respect to the X-ray beam (which is parallel to the long edge of the glass substrate) is calculated. In Eq. (1) we use the fact that the Ewald sphere has a finite curvature, the wave vector component in beam direction $q_{x}$ is nonzero! Due to the tilt and due to the interference of the scattered waves induced by the lateral structure the two components $q_{x}, q_{y}$ of the wave vector transfer parallel to the sample surface are coupled. The virtual, enlarged period stems from the tilt of the nanochannel array with respect to the beam.

Consequently, GISAXS allows for the determination of the alignment of the channel array, yielding a value of $\omega=-6.4^{\circ}$. This orientational information, important for sev- eral applications, is inaccessible with optical microscopy or AFM, as can seen from Fig. 1.

In summary, with an extremely simple deposition process, based on orienting dewetting structures by mechanical wiping, PDMS nanochannels are prepared on glass. As demonstrated with GISAXS, being contact-free, operating under air and requiring no special sample preparation, the extremely shallow nanochannels are well aligned on large scale surface areas of several square millimeters and the orientation is nearly matching the edges of the glass substrate used $\left(6.4^{\circ}\right.$ mismatch of the orientation only). Thus well controlled surface patterns result from this easy preparation under the mechanical and ambient conditions mentioned earlier, which might be interesting for future applications in micro- and nanofluidics.

The authors thank M. Dommach and R. Döhrmann for their help with setting up the GISAXS experiment. This work was financially supported by the DFG Schwerpunktprogramm SPP 1164 "Nano- and Microfluidics" (Mu1487/2).

${ }^{1}$ L. Peters, Semicond. Int. 38, 28 (2005).

${ }^{2}$ Z. Yu and S. Y. Chou, Nano Lett. 4, 341 (2004).

${ }^{3}$ A. Fahmi and M. Stamm, Langmuir 21, 1062 (2005).

${ }^{4}$ Q. Lu and C. G. Bazuin, Nano Lett. 5, 1309 (2005).

${ }^{5}$ T. Albrecht, J. Schotter, G. A. Kästle, N. Emley, T. Shibauchi, L. KrusinElbaum, K. Guarini, C. T. Black, M. T. Tuominen, and T. P. Russell, Science 290, 2126 (2000).

${ }^{6}$ X. Yan, F. Liu, Z. Li, and G. Liu, Macromolecules 34, 9112 (2001).

${ }^{7}$ J. P. Spatz, M. Möller, M. Noeske, R. J. Behm, and M. Pietralla, Macromolecules 30, 3874 (1997).

${ }^{8}$ E. Bormashenko, R. Pogreb, O. Stanevsky, Y. Bormashenko, T. Stein, V.-Z. Gaisin, R. Cohen, and O. V. Gendelman, Macromol. Mater. Eng. 290, 114 (2005).

${ }^{9}$ L. J. Guo, X. Cheng, and C. F. Chou, Nano Lett. 4, 69 (2004).

${ }^{10}$ C. Lee, E. H. Yang, N. V. Myung, and T. George, Nano Lett. 3, 1339 (2003).

${ }^{11}$ N. R. Tas, J. W. Berenschot, P. Mela, H. V. Jansen, M. Elwenspoek, and A. van den Berg, Nano Lett. 2, 1031 (2002).

${ }^{12}$ S. O. Kim, H. H. Solak, M. P. Stoykovich, N. J. Ferrier, J. J. de Papblo, and P. F. Nealey, Nature (London) 424, 411 (2003).

${ }^{13}$ T. Xu, J. T. Goldbach, and T. P. Russell, Macromolecules 36, 7296 (2003).

${ }^{14}$ A. V. Kyrylyuk, A. V. Zvelindovsky, G. J. A. Sevink, and J. G. E. M. Fraaije, Macromolecules 35, 1473 (2002).

${ }^{15}$ C. Tang, A. Tracz, M. Kruk, R. Zhang, D.-M. Smilgies, K. Matyjaszewski, and T. Kowalewski, J. Am. Chem. Soc. 127, 6918 (2005).

${ }^{16}$ S. Kubowicz, A. F. Thunemann, T. M. Geue, U. Pietsch, M. D. Watson, N. Tchebotareva, and K. Müllen, Langmuir 19, 10997 (2003).

${ }^{17}$ KIMWIPES Lite, Kimberly Clark.

${ }^{18}$ P. Müller-Buschbaum, J. Phys.: Condens. Matter 15, R1549 (2003).

${ }^{19}$ I. Karapanagiotis, D. F. Evans, and W. W. Gerberich, Colloids Surf., A 207, 59 (2002).

${ }^{20}$ P. Müller-Buschbaum, E. Bauer, O. Wunnicke, and M. Stamm, J. Phys.: Condens. Matter 17, S363 (2005).

${ }^{21}$ P. Müller-Buschbaum, Anal. Bioanal. Chem. 376, 3 (2003).

${ }^{22}$ T. Salditt, T. H. Metzger, J. Peisl, and G. Goerigk, J. Phys. D 28, A236 (1995).

${ }^{23}$ T. Salditt, H. Rhan, T. H. Metzger, J. Peisl, R. Schuster, and J. P. Kotthaus, Z. Phys. B: Condens. Matter 96, 227 (1994).

${ }^{24}$ D. Le Bolloch, F. Livet, F. Bley, T. Schulli, M. Veron, and T. H. Metzger, J. Synchrotron Radiat. 9, 258 (2002). 